\title{
Community Perceptions on Integrating Animal Vaccination and Health Education by Veterinary and Public Health Workers in the Prevention of Brucellosis among Pastoral Communities of South Western Uganda
}

\author{
Catherine Kansiime*1, Lynn M. Atuyambe ${ }^{1}$, Victor Guma ${ }^{1}$, Anthony Mugisha ${ }^{1}$, Samuel \\ Mugisha $^{1}$, Benon B. Asiimwe' ${ }^{1}$, Innocent B. Rwego ${ }^{2}$ and Elizeus Rutebemberwa ${ }^{1}$
}

${ }^{1}$ Health Policy Planning and Management, Makerere University, Kampala, Uganda; ${ }^{2}$ University of Minnesota, Minnesota, MN, USA

\section{Objective}

To explore community perceptions on integration of animal vaccination and health education by veterinary and public health workers in the management of brucellosis.

\section{Introduction}

Brucellosis is a zoonotic disease of veterinary, public health and economic significance in most developing countries, yet there are few studies that show integrated human and veterinary health care intervention focusing on integration at both activity and actors levels. The aim of our study, therefore, was to explore community perceptions on integration of animal vaccination and health education by veterinary and public health workers in the management of brucellosis.

\section{Methods}

This study used a qualitative design where six Focus Group Discussions (FGDs) that were homogenous in nature were conducted, two from each sub-county one with the local leaders, and another with pastoralists and farmers. Five Key Informant Interviews (KIIs) with two public health workers and three veterinary officers from three sub-counties in Kiruhura district were conducted. All FGDs were conducted in the local language and tape recorded with consent from the participants. KIIs were in English and later transcribed and analyzed using latent content data analysis method.

\section{Results}

All the groups mentioned that they lacked awareness on brucellosis commonly known as 'brucella' and its vaccination in animals. Respondents perceived improvement in human resources, facilitation of the necessary activities such as sensitization of the communities about brucellosis, and provision of vaccines and diagnostic kits as important in the integration process in the communities. The Focus Group Discussion participants also believed that community participation was crucial for sustainability and ownership of the integration process.

\section{Conclusions}

The study reported a limited understanding of brucellosis and its vaccination in animals. The community members believed that mass animal vaccination in combination with health education about the disease is possible if it involves government and all other stakeholders such as wildlife authorities, community members, local to national political leaders, as well as the technical personnel from both veterinary, medical and public health sectors.

\section{Keywords}

Brucellosis; Perceptions; Integration; Animal Vaccination; Health Education

\section{Acknowledgments}

The authors are grateful for financial support from International Development Research Centre (Canada), all the interview respondents who participated in the study, the community leaders and to all the research assistants for their contribution to the success of this research.

*Catherine Kansiime

E-mail: cathie.kansiime@gmail.com 